\title{
Rabl's Model of the Interphase Chromosome Arrangement Tested in Chinese Hamster Cells by Premature Chromosome Condensation and Laser-UV-Microbeam Experiments
}

\author{
T. Cremer ${ }^{1}$, C. Cremer ${ }^{2}$, H. Baumann ${ }^{1,4}$, E.-K. Luedtke ${ }^{3}$, K. Sperling ${ }^{3}$, V. Teuber ${ }^{2.5}$, and C. Zorn ${ }^{2}$ \\ ${ }^{1}$ Institut für Anthropologie und Humangenetik der Universität Heidelberg, Im Neuenheimer Feld 328, D-6900 Heidelberg, \\ Federal Republic of Germany \\ ${ }^{2}$ Institut für Humangenetik und Anthropologie der Universität Freiburg i. Br., Albertstraße 11, D-7800 Freiburg, Federal Republic of Germany \\ ${ }^{3}$ Institut für Humangenetik der Freien Universität Berlin, Heubnerweg 6, D-1000 Berlin 19, Federal Republic of Germany
}

Summary. In 1885 Carl Rabl published his theory on the internal structure of the interphase nucleus. We have tested two predictions of this theory in fibroblasts grown in vitro from a female Chinese hamster, namely (1) the Rabl-orientation of interphase chromosomes and (2) the stability of the chromosome arrangement established in telophase throughout the subsequent interphase. Tests were carried out by premature chromosome condensation (PCC) and laser-UV-microirradiation of the interphase nucleus. Rabl-orientation of chromosomes was observed in G1 PCCs and G2 PCCs. The cell nucleus was microirradiated in Gl at one or two sites and pulse-labelled with ${ }^{3} \mathrm{H}$-thymidine for $2 \mathrm{~h}$. Cells were processed for autoradiography either immediately thereafter or after an additional growth period of 10 to $60 \mathrm{~h}$. Autoradiographs show unscheduled DNA synthesis (UDS) in the microirradiated nuclear part(s). The distribution of labelled chromatin was evaluated in autoradiographs from 1035 cells after microirradiation of a single nuclear site and from 253 cells after microirradiation of two sites. After 30 to $60 \mathrm{~h}$ postincubation the labelled regions still appeared coherent although the average size of the labelled nuclear area fr increased from $14.2 \%(0 \mathrm{~h})$ to $26.5 \%(60 \mathrm{~h})$. The relative distance $\mathrm{dr}$, i.e. the distance between two microirradiated sites divided by the diameter of the whole nucleus, showed a slight decrease with increasing incubation time. Nine metaphase figures were evaluated for UDS-label after microirradiation of the nuclear edge in G1. An average of 4.3 chromosomes per cell were labelled. Several chromosomes showed joint labelling of both distal chromosome arms including the telomeres, while the centromeric region was free from label. This label pattern is interpreted as the result of a V-shaped orientation of these particular chromosomes in the interphase nucleus with their telomeric regions close to each other at the nuclear edge. Our data support the tested predictions of the Rabl-model. Small time-dependent changes of the nuclear space

\footnotetext{
4 Part of this work is included in the doctoral thesis of H. Baumann to be submitted to the Faculty of Biology of the University of Heidelberg

5 Part of this work is included in the doctoral thesis of V. Teuber to be submitted to the Faculty of Medicine of the University of Freiburg i. $\mathrm{Br}$.

Offprint requests to: T. Cremer
}

occupied by single chromosomes and of their relative positions in the interphase nucleus seem possible, while the territorial organization of interphase chromosomes and their arrangement in general is maintained during interphase. The present limitations of the methods used for this study are discussed.

\section{Introduction}

In 1885 Carl Rabl published his theory on the internal structure of the interphase nucleus. This theory was based on microscopic examination of Salamandra maculata and Proteus cells. It states-in modern terminology-that chromosomes maintain their individuality as well as their anaphase-telophase orientation throughout interphase and occupy distinct territories in the interphase nucleus. These paradigms were then much more firmly established by the work of Boveri using Ascaris megalocephala (Boveri 1888, 1909). Boveri also showed clearly that the telomeres of the chromosomes end at the nuclear envelope. In Rabl's original drawing of his model the place and the orientation of each interphase chromosome is given by the course of a primary nuclear thread, from which secondary and tertiary threads extend as lateral projections and form the three dimensional chromatin network of the interphase nucleus (Fig. 1a,b). The centromeres are preferentially located on one site of the nucleus, which Rabl calls the "Polfeld". From this Polfeld the primary threads run to the opposite site of the nucleus, called the "Gegenpolseite", mainly along the periphery of the nucleus, but occasionally also directly through the interior of the cell nucleus. Telomeres are associated with each other at the Gegenpolseite. Due to the assumed V-shape of the primary threads of metacentric chromosomes the telomeres of both arms are closely associated. In prophase the extended chromatin streams back into the primary threads along pathways preformed by the secondary and tertiary threads and mitotic chromosomes become visible as separate entities (Fig. 1c-f). 


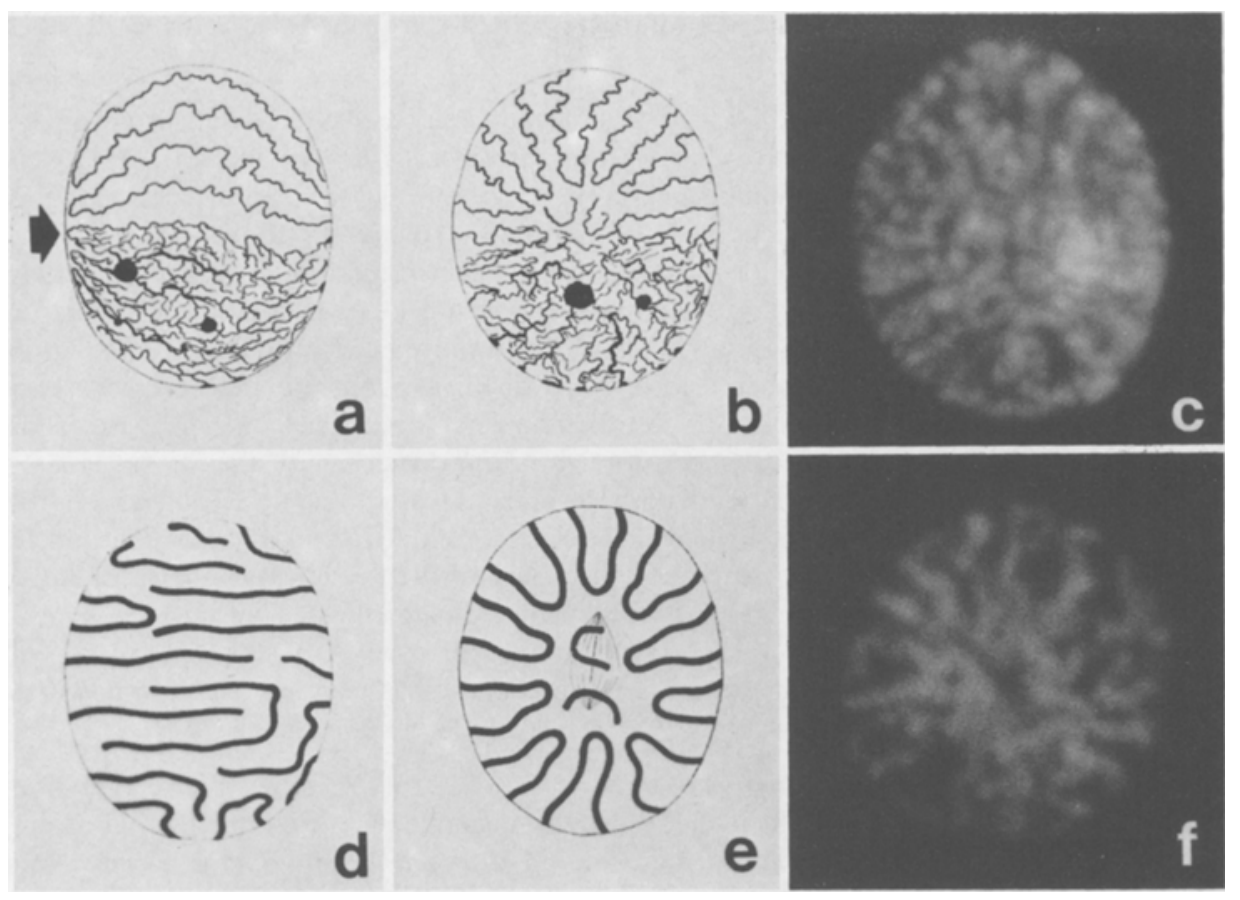

Fig. 1 a-e. Rabl's scheme of the interphase nucleus reprinted from (Rabl 1885, Table XII, 12a, b); a nucleus seen from the side, arrow indicates the "Polfeld", b the same nucleus seen from another position with the "Polfeld" in the centre. The upper part of the nucleus shows the course of Rabl's "primäre Kernfäden" (primary threads). Note that the centromeres are cooriented at the "Polfeld". The lower part represents Rabl's "Kernnetz" (nuclear network), which is produced by numerous lateral projections originating from the primary threads. $\mathbf{c}$ and $\mathbf{f}$ Fluorescence microscopy showing early prophase (c) and a somewhat later stage of prophase (f) of an unfixed Chinese hamster cell nucleus stained with Hoechst 33258 $(15 \mu \mathrm{g} / \mathrm{ml}$ in Hanks' solution at room temperature, two washes with Hanks' for at least 5 min each to remove excess of the stain). These stages approximately represent Rabl's "dichtes Knäuel" (dense coil) (c) and "lockeres Knäuel" (loose coil) (f). d and e "Lockeres Knäuel" drawn from the "Gegenpolseite" (d) and from the "Polseite" (e) reprinted from Rabl (1885, Table XII, 2b, c). Note that the orientation of the interphase chromosomes as indicated by primary threads in $\mathbf{a}$ and $\mathbf{b}$ is maintained in prophase chromosomes. In comparison with Rabl's dense coils chromosomes in loose coils appear thicker and shorter. For further explanation of Rabl's model see text

Astonishingly since then our understanding of the internal order of the interphase nucleus has little improved. Still the question of the actual arrangement of the interphase chromosomes and their possible functional significance is a matter of considerable controversy at least in mammalian cells (Vogel and Schroeder 1974; Comings 1980; for plant cells see Avivi and Feldman 1980). In the case of chromosome territories as proposed by the Rabl-Boveri model, the assumed fixing points of each chromosome to the nuclear matrix and the nuclear membrane (Vogel and Schroeder 1974; Comings 1980) should be concentrated within a small and coherent nuclear segment. In other models which consider a more extended distribution of chromosomes within the nucleus, these fixing points should be scattered over a much larger area of the nuclear envelope and matrix as well (Comings 1968; Vogel and Schroeder 1974). Evidently this latter type of organization would result in more intermingling of the chromatin fibres of different chromosomes. Evidence for a territorial organization has recently been reviewed by Comings (1980). However, the possible dynamics of chromatin movements during interphase, especially during S-phase, are still far from clear. Vogel and Schroeder (1974) have discussed the possibility that somatic pairing may not exist during the whole interphase, but in part of it only. Such a dynamic view of the interphase arrangement is in contrast to Boveri's conclusion that the interphase is, with regard to chromosome configuration, the most conservative phase (Boveri 1909 , p 208). We may, however, consider the possibility that certain major fixing points, for example at the nuclear envelope, are maintained during interphase, while others are changing when the cell proceeds from G1 through S-phase. If so, the chromatin fibre representing an individual chromosome might be localized in the same distinct territory in telophase and the following prophase as shown by Boveri (1909), but much more intermingled with the fibres from other chromosomes in S-phase as compared with early $\mathrm{G} 1$ and late G2. The decondensation-condensation cycle of interphase chromosomes as revealed by PCC experiments (Sperling and Rao 1974) would fit such an idea.

The scarcity of evidence regarding the interphase chromosome arrangement is obviously due to the fact that individual chromosomes in most cell types cannot be seen directly in the interphase nucleus. The Rabl-Boveri model was therefore based mainly on indirect conclusions drawn from observations of chromosomes in fixed mitotic cells. In the meantime the arrangement of chromosomes has been extensively studied in metaphase preparations (compare Zang and Back 1968; Zankl and Zang 1974; Hens et al. 1975; Kirsch-Volders et al. 1977, 1980), but direct approaches to study the topography of chromatin in the interphase nucleus have remained very limited (Hoehn and Martin 1973; Comings 1980). Only recently have methods become available which can be used to study the problem of the internal order of chromosomes in the mammalian interphase nucleus in a straightforward way instead of drawing indirect conclusions from studies of the arrangement of chromosomes at metaphase (Vogel and Schroeder 1974; Cremer et al. 1979 a, 1980; Sperling and Luedtke 1981; Hager et al. to be published). At the same time these studies provide possibilities for testing whether the metaphase arrangement of 
chromosomes reflects the interphase arrangement as well (Cremer et al. in preparation). In the present investigation we have applied two methods which we consider as especially promising tools for elucidating the arrangement of chromosomes in the interphase nucleus, namely premature chromosome condensation (PCC) (Sperling and Luedtke 1981) and microirradiation of the nucleus (Cremer et al. 1979a, 1980; Zorn et al. 1979). Evidence for chromosome territories in the Chinese hamster G1-nucleus has previously been established by microirradiation experiments (Cremer et al. 1979a; Zorn et al. 1979). In the present investigation we have tested the validity of two other predictions of the Rabl-Boveri model, namely the orientation of the interphase chromosomes and the stability of the chromosome arrangement once established at telophase throughout the subsequent interphase. The object of our investigation was the nucleus of Chinese hamster fibroblasts cultured in vitro. These cells contain 22 chromosomes and should allow a good comparison with Salamandra maculata, for which Rabl stated a number of 24 chromosomes.

\section{Materials and Methods}

\section{Cell Strain and Culture Conditions}

A fibroblastoid Chinese hamster cell strain was established from lung tissue of a three-week-old female and grown under standard conditions (Zorn et al. 1979). A number of early passage cultures were stored in liquid nitrogen and cells from passage numbers $10-15$ were used for the experiments. At this passage level the majority of cells had still maintained their diploid status as shown by Q-banding analysis.

\section{Premature Chromosome Condensation}

Unsynchronized Chinese hamster cells were fused to mitotic HeLa cells for the induction of premature chromosome condensation (PCC). The detailed procedure for PCC induction has been described earlier (Sperling and Rao 1974). In brief: mitotic HeLa cells and Chinese hamster interphase cells were mixed in a ratio of $2: 1\left(1 \times 10^{6}\right.$ cells total $)$ and incubated with $500 \mathrm{HAU}$ of UV inactivated Sendai virus at $4^{\circ} \mathrm{C}$ for $15 \mathrm{~min}$. The virus-cell mixture was then transferred into a water bath of $37^{\circ} \mathrm{C}$ for $40 \mathrm{~min}$ and thereafter treated with hypotonic $\mathrm{KCl}(0.075 \mathrm{M})$ for $20 \mathrm{~min}$. The cells were then centrifuged and carefully fixed by layering a mixture of methanol/acetic acid (3:1) on top of the cell sediment. Thirty minutes later the contents of the centrifuge were pipetted. After three changes of fixative the cells were dropped onto wet slides, air-dried, and stained with Giemsa.

Laser Microbeam. The laser microbeam has been described earlier (Cremer et al. 1974, 1976). Briefly, a continuous wave coherent UV-beam of wavelength $257 \mathrm{~nm}$ was focused to one site in a cell with a quartz microscope objective (Zeiss Ultrafluar $32 \times / 0.40 \mathrm{Ph}$ Glyz.) which was simultaneously used for observation of the cells in phase contrast. Aiming was performed by means of a cross hair. The diameter of the microbeam at its focal site is approximately $1 \mu \mathrm{m}$. By changing the position of an adapting lens, different diameters of the irradiation field in the object plane can be adjusted (Cremer et al. 1981). In the present experiments roughly 10 to $15 \%$ of the total area of nuclei of average size was microirradiated. The UV power incident at the cell surface was approximately $7.5 \times 10^{-9} \mathrm{~W}$, the irradiation time varied between $1 / 125$ and $1 / 15$ of a second.

Conditions of Microirradiation. Cells were synchronized by selective harvest of mitotic cells and incubated in Eagle's minimum essential medium with $0.5 \%$ foetal calf serum (FCS) to keep them within the Gl phase of the cell cycle for the desirable period of time. Experimental fields of approximately $0.25 \mathrm{~mm}^{2}$ were marked by scalpel cuts in the bottom of plastic petri dishes. Petri dishes were placed into special irradiation chambers and cells growing in experimental fields were used for microirradiation experiments, while cells outside these fields served as controls. In some experiments the site of microirradiation within the nucleus was chosen at random except for the fact that nucleoli were excluded from microirradiation. In other experiments the edge of the nucleus was microirradiated at one site, or both poles of the ellipsoid nuclei were microirradiated close to the nuclear edge. A detailed description of the microirradiation procedure has been presented in previous publications (Cremer et al. 1976; Zorn et al. 1979).

Posttreatment of Microirradiated Cells. After microirradiation of cells in G1, cells were incubated for two hours with medium without FCS containing $10 \mu \mathrm{Ci} / \mathrm{ml}^{3} \mathrm{H}$-thymidine (Amersham, sp.act. $47 \mathrm{Ci} / \mathrm{mmol}$ ). Thereafter the cells were either fixed immediately or allowed to proceed through the cell cycle for different periods of time after feeding them with fresh medium without ${ }^{3} \mathrm{H}$-thymidine and containing $15 \%$ FCS (postincubation period). Interphase cells were fixed with $3 \%$ glutaraldehyde for $10 \mathrm{~min}$ and five subsequent changes of $2 \%$ perchloric acid. Mitotic cells were blocked by addition of $1 \mu \mathrm{g} / \mathrm{ml}$ colchicine for three hours. Chromosome preparations were performed in situ as previously described (Zorn et al. 1979) and stained with DAPI (Schnedl et al. 1977). Autoradiography was carried out following standard procedures and autoradiographs were slightly stained with acetic orceine (Zorn et al. 1979).

\section{Results}

\section{Premature Chromosome Condensation Experiments}

Premature chromosome condensation (PCC) was used to analyze the arrangement of Chinese hamster chromosomes in the interphase nucleus with respect to polarity. PCCs were classified according to the polarization of chromosomes as first described by Rabl in 1885 (Rabl orientation). A completely polarized orientation was assumed when almost all chromosomes (at least more than two thirds, i.e. more than 15 out of the total number of 22 chromosomes) exhibited a polar orientation with the centromeres coorientated to each other, and the metacentric and submetacentric chromosomes appeared more or less V-shaped and the acrocentric chromosomes rod-shaped (Fig. 2). Occasionally the plates exhibited two centres instead of one (Fig. 2 a). If only about half of the chromosomes (some 10 to 15$)$ were oriented in this way the plates were classified as "partially polarized". In the case of more or less random orientation they were classified as "non-polarized". The results of three independent fusion experiments are shown in Table 1. The frequencies of completely and partially polarized PCCs varied between $28 \%$ and $72 \%$. It is important that the polarized chromosome arrangement was observed both in G1 and G2 PCCs. We noted somewhat higher frequencies in G2 PCCs as compared to G1 PCCs. This difference can probably be 


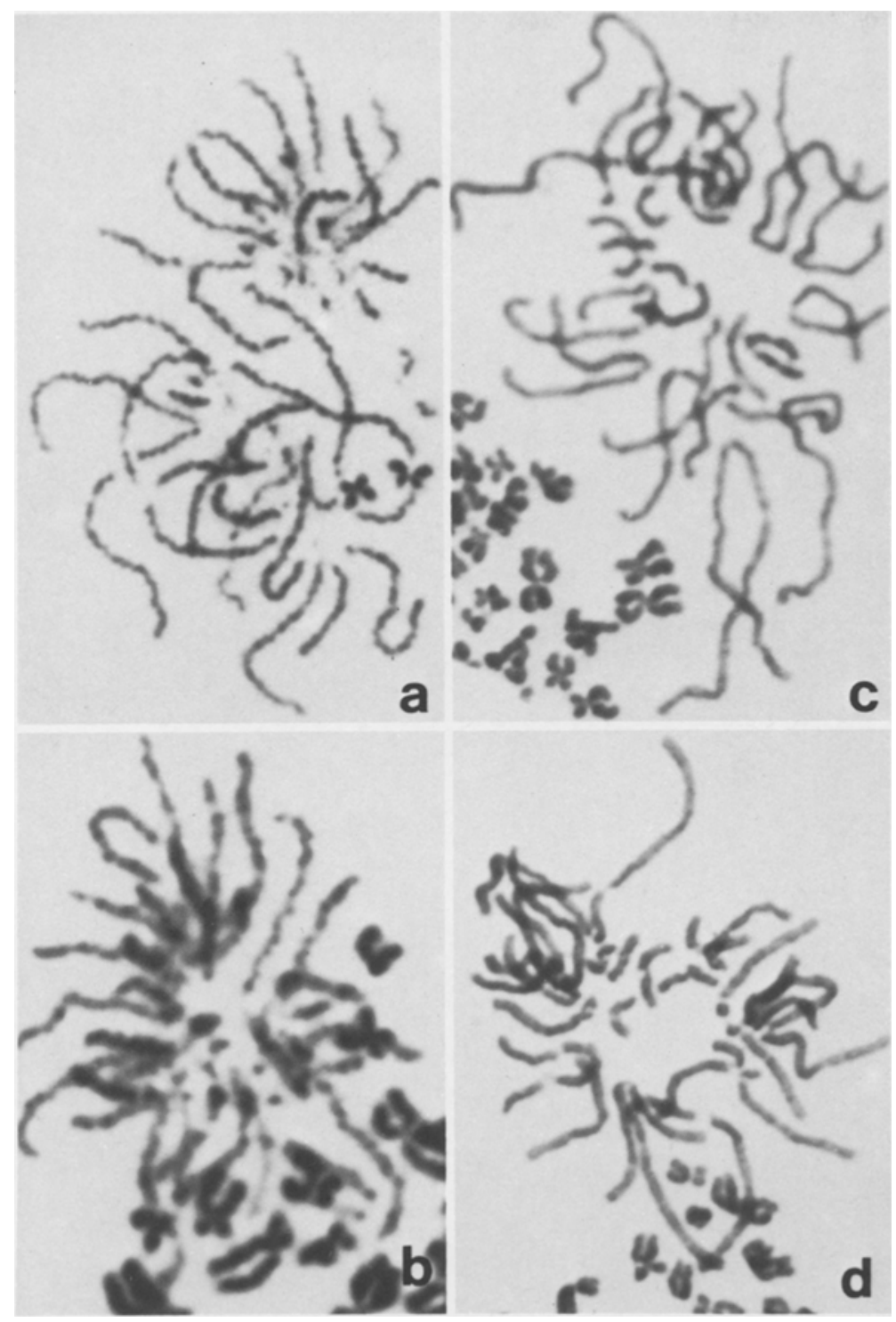

Fig. 2a-d. Orientation of prematurely condensed chromosomes of Chinese hamster nuclei after fusion with mitotic HeLa cells; $\mathbf{a}$ and b completely polarized G1 PCC, c and $\mathbf{d}$ completely polarized G2 PCC. Note that almost all centromeres are coorientated, exhibiting the "Rabl orientation". In a two centres are visible
Table 1. Orientation of prematurely condensed chromosomes

\begin{tabular}{lllll}
\hline $\begin{array}{l}\text { Exp. } \\
\text { no. }\end{array}$ & $n$ & $\begin{array}{l}\text { Completely } \\
\text { polarized }\end{array}$ & $\begin{array}{l}\text { Partially } \\
\text { polarized }\end{array}$ & Nonpolarized \\
\hline 1 & 100 & $36 \%$ & $36 \%$ & $28 \%$ \\
2 & 100 & $9 \%$ & $37 \%$ & $54 \%$ \\
3 & 50 & $6 \%$ & $22 \%$ & $72 \%$ \\
\hline
\end{tabular}

$n$ : Number of evaluated G1- and G2-PCCs

attributed to the rather lengthy G1 PCC chromosomes which make the observation of a polarized orientation more difficult. Our PCC experiments are in agreement with Rabl's model of a polarized arrangement of interphase chromosomes. The experiments are also compatible with Rabl's and Boveri's arguments that the arrangement of chromosomes established in telophase is maintained during interphase. Independent evidence for these conclusions will be described in the next section.

\section{Microbeam Experiments}

Experimental Rationale. The following microbeam experiments were performed to answer two questions: (1) Do G1-chromosomes exhibit a polarized orientation as suggested by PCCexperiments? (2) Does the G1-arrangement of chromosomes change when the cell proceeds through the cycle?

The experimental rationale of the microbeam approach for answering these questions can easily be understood by comparing the interphase nucleus and its chromosomes with a coil composed of a number of threads. Figure 3 shows two basically different arrangements of four threads. Each thread may form a distinct territory (coil A) (Fig. 3a) or the threads may be extended throughout the whole coil (coil B) (Fig. 3d). When all threads are of the same colour it becomes difficult or even impossible to visualize the individual arrangement of each thread in the coil. To overcome this problem a small segment of the coil is marked by some colour (Fig. 3b, e). If the arrangement of threads in the coil is static, then the colour should remain concentrated thereafter at the marked segment. Furthermore, if two different segments are marked, the distance 

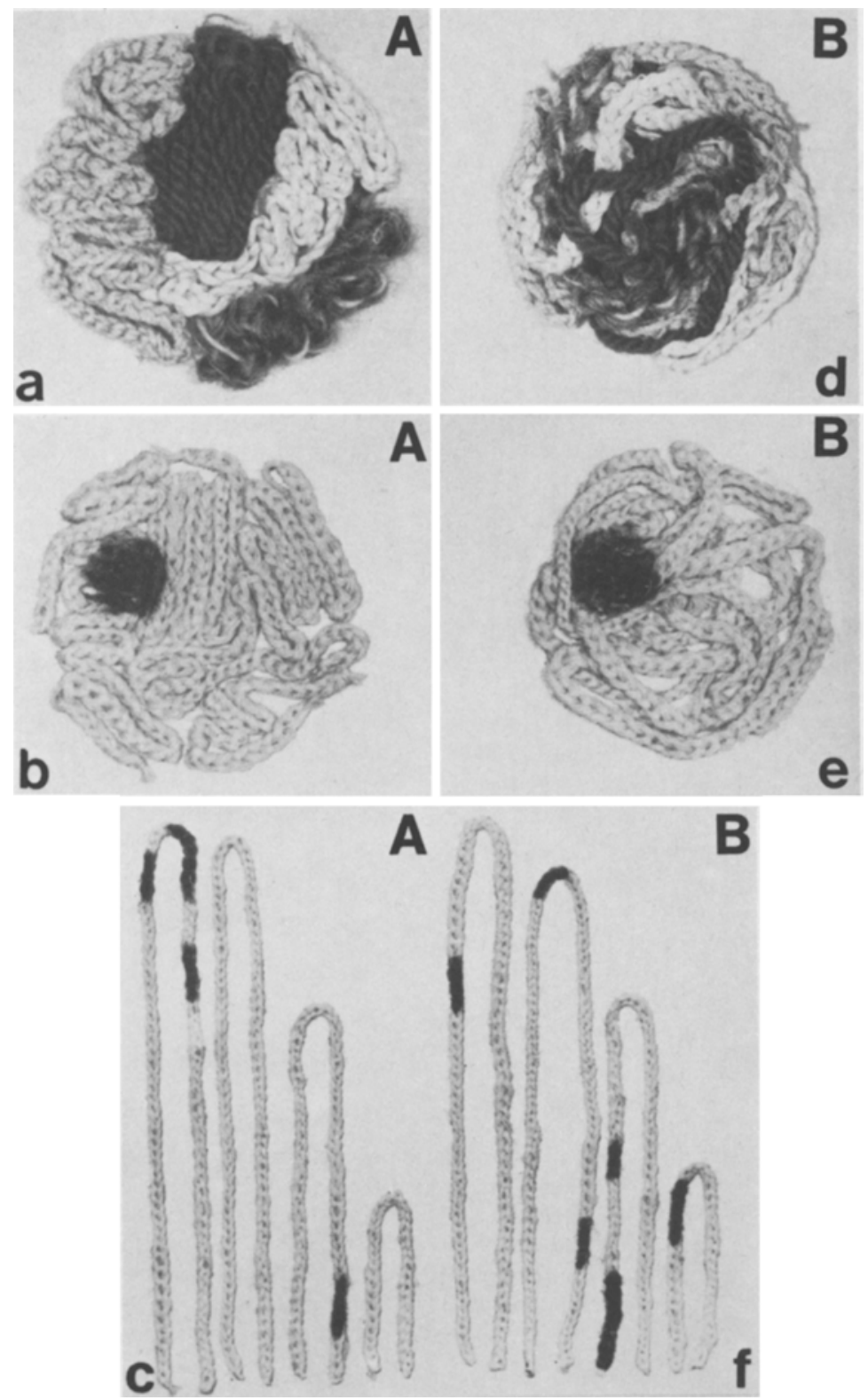

Fig. 3a-f. Illustration of the experimental rationale of microirradiation experiments: the interphase nucleus is compared with a coil composed of a number of threads (chromosomes). $\mathbf{a}$ and $\mathbf{b}$ Two basically different arrangements A and B. In A each thread forms a distinct territory (domain), in B the threads are extended throughout the whole coil. The examples shown here are meant to illustrate the power of the microbeam method to decide between different types of arrangements; they are not intended to suggest an actual chromosome arrangement of any particular cell type. $\mathbf{b}$ and e Similar arrangements of threads as shown in a and $\mathbf{d}$. All threads are of the same light colour so that it becomes difficult to distinguish the individual arrangement of each thread in the coil. A small part is marked by some black colour. In microirradiation experiments an autoradiographic procedure is used to mark the irradiated nuclear site (compare Fig.4). $\mathbf{c}$ and $\mathbf{f}$ The same threads as shown in $\mathbf{b}$ and $\mathbf{e}$ after disassembling of the two coils. Marked regions of the individual threads can be identified. Three sources of information can be used to reconstruct the original arrangement of threads: (1) the site of the coil selected for marking, (2) the number of marked and unmarked threads, (3) the distribution of markings along the individual threads

between these segments should not change with time. On the other hand, if the arrangement of threads changes then we would expect a time dependent effect on the distribution of the colour and the distance between two coloured segments. Finally, if coils with one marked segment are disassembled into individual threads three sources of information can be used to reconstruct the arrangement of threads at the time of segmentmarking: (a) the site of the coil selected for marking, (b) the number of marked threads, and (c) the distribution of markings along individual threads. In the case of a nonterritorial organization as exemplified in coil B the markings are more or less scattered over many or even all the threads (Fig. $3 \mathrm{f}$ ). In contrast, a territorial organization would result in extensive markings on a few threads, while other threads representing the majority of threads in the case of larger numbers would remain completely untouched (Fig. $3 \mathrm{c}$ ). If all the threads end at the periphery of the coil then marking of a peripheral segment should result in threads stained at their ends.

This rationale was experimentally realized as follows. Chinese hamster cells were microirradiated in G1 at one or two sites and pulse-labelled with ${ }^{3} \mathrm{H}$-thymidine for $2 \mathrm{~h}$ immediately thereafter. ${ }^{3} \mathrm{H}$-thymidine was incorporated into the damaged DNA 
strands during unscheduled DNA synthesis (UDS) in the microirradiated nuclear part. Cells were processed for autoradiography either immediately or after they had proceeded through additional parts of the cell cycle and in some cases reached the first post-irradiation mitosis.

Static or Dynamic Arrangement of Chromosomes During Interphase? Figure 4 shows nuclei microirradiated either at a random site of the nucleoplasm or at both poles some $2 \mu \mathrm{m}$ inside from the nuclear edge. The autoradiographs were obtained immediately after the labelling (Fig. $4 \mathrm{a}, \mathrm{c}$ ) or after an additional postincubation period of 30 and $60 \mathrm{~h}$ (Fig. 4b,d). Notably, all these nuclei show label restricted to one and two sites, respectively. After 30 to $60 \mathrm{~h}$ postincubation the enlarged nuclei often showed a less dense accumulation of silver grains over the irradiated nuclear part as compared with cells processed for autoradiography immediately after the labelling period. However, the labelled regions still appeared rather coherent even after such prolonged postincubation periods. In parallel sets of experiments microirradiated cells were treated in exactly the same way except for an additional continuous labelling with ${ }^{3} \mathrm{H}$-thymidine $(0.1 \mu \mathrm{Ci} / \mathrm{ml})$ for the whole growth period. In these experiments roughly $60 \%$ of the nuclei became labelled over their whole area after $40 \mathrm{~h}$, indicating that a large fraction of these cells had proceeded into S-phase (Fig. 5).

For quantitative analysis camera lucida drawings of single microirradiated nuclei were obtained at a convenient magnification. As shown in Fig. 6 the following measurements were carried out. The UDS-labelled nuclear area $f$ was arbitrarily encircled and both $f$ and the total nuclear area $F$ were determined by planimetry. In addition we measured the distance $d$ between the centres of two labelled nuclear sites and the diameter $D$ of the nucleus given by a line through the two centres. When comparing $\mathrm{f}$ and $\mathrm{d}$ for different postincubation periods $(0$ to $60 \mathrm{~h})$ one has to take into account the increase of the nuclear size. The mean values for $\mathrm{F}\left(\mu \mathrm{m}^{2}\right)$ at $0(n=361)$ and $20(n=215)$ hours postincubation did not significantly differ from each other in the $t$-test $(179.1 \pm 3.93$ SEM versus $187.4 \pm$ $4.61)$. Between 20 and $60 \mathrm{~h}$ a significant increase $(P<0.001)$ of $\mathrm{F}$ was noted by linear regression analysis with a regression co-

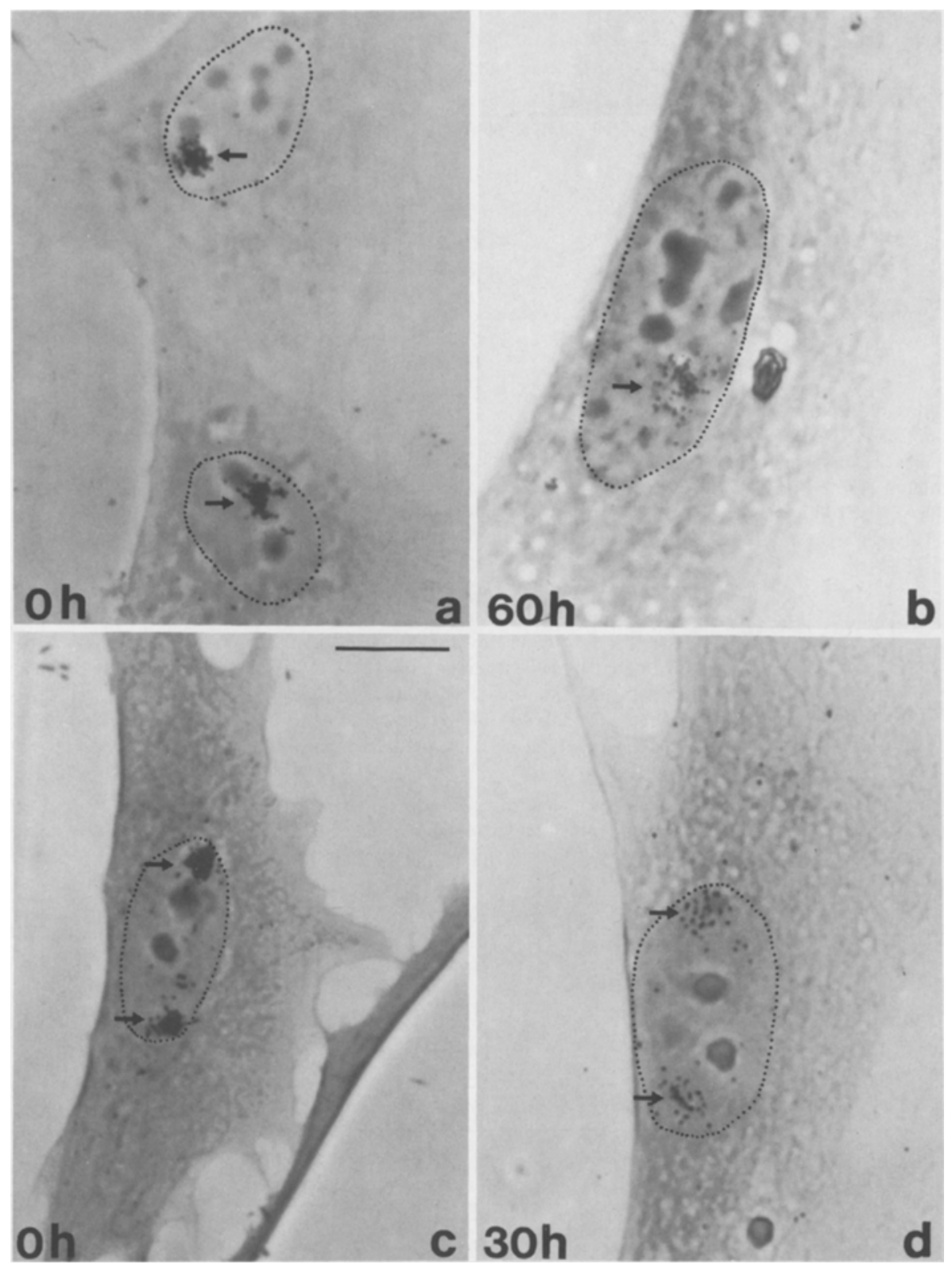

Fig. 4. Autoradiographs of Chinese hamster cells after UV-microirradiation of the nucleus in $\mathrm{Gl}$ at one site (a and $\mathbf{b}$ ) or at two sites (c and d). Irradiated cells were labelled with ${ }^{3} \mathrm{H}$-thymidine for $2 \mathrm{~h}$ while unscheduled DNA synthesis (UDS) was taking place at the microirradiated site(s) and fixed either immediately after the label period $(a, c)$ or after an additional postincubation period of $30 \mathrm{~h} \mathrm{(d)} \mathrm{and} 60 \mathrm{~h}(\mathrm{~b})$ respectively. Bar, $10 \mu \mathrm{m}$ 


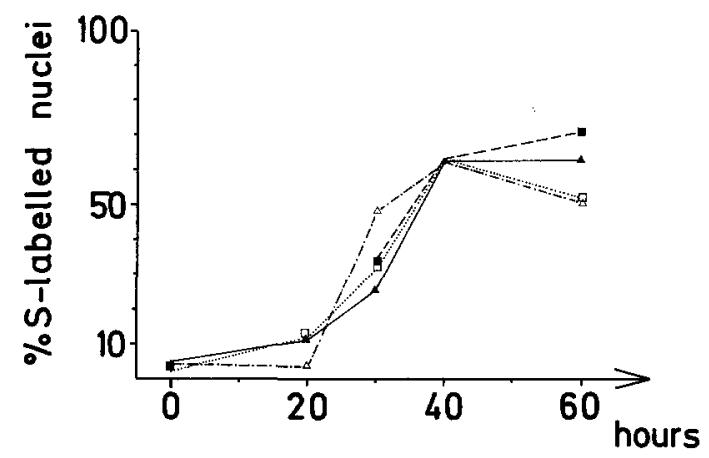

Fig. 5. Entry of microirradiated cells into S-phase. Nuclei were microirradiated in G1 at one site with different doses and pulse-labelled with ${ }^{3} \mathrm{H}$-thymidine for two hours as described for the detection of unscheduled DNA synthesis (UDS). Thereafter the cells were allowed to proceed through the cycle by adding fresh medium with $15 \%$ FCS containing $0.1 \mu \mathrm{Ci} / \mathrm{ml}^{3} \mathrm{H}$-thymidine and processed for autoradiography after different postincubation periods. Nuclei with label distributed over their whole area were considered as having entered S-phase during the postincubation time. The majority of nuclei still considered in G1 showed label restricted to the microirradiation site indicating UDS (compare Fig. 4). $\Delta-\Delta$ : No irradiation, $0 \mathrm{~h}$ (127 nuclei), $20 \mathrm{~h}$ (496),

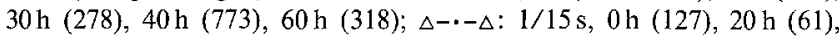

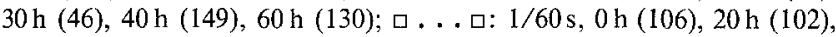
$30 \mathrm{~h}(151), 40 \mathrm{~h}(256), 60 \mathrm{~h}(80)$; $-\cdots: 1 / 125 \mathrm{~s}, 0 \mathrm{~h}(181), 20 \mathrm{~h}(0)$,

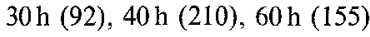

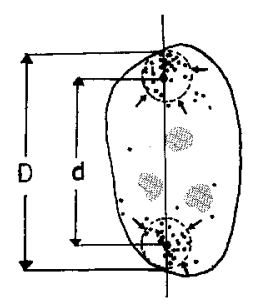

Fig. 6. Measurements performed in autoradiographs of single microirradiated nuclei. The scheme shows a nucleus microirradiated at two sites (compare Fig. 4d). F: Total nuclear area; f: labelled nuclear area per microirradiation site (small arrows); D: diameter of the nucleus; d: distance of the centres of the two labelled sites; $f r=f / F \times 100$ : percent UDS-labelled area; $\mathrm{dr}=\mathrm{d} / \mathrm{D}$ : relative distance between two microirradiated sites

efficient of +0.23 . The mean value for $F\left(\mu \mathrm{m}^{2}\right)$ was $236.4 \pm$ $7.19(\mathrm{SEM})$ after $40 \mathrm{~h}(n=223)$ and $312.0 \pm 11.77$ after $60 \mathrm{~h}$ $(n=171)$. This increase presumably reflects the S-phase dependent increase of the nuclear volume, while mitotic events were rare in these cells. To compensate for the increase of the nuclear size we calculated (a) the relative labelled nuclear area fr as percent of the total nuclear area $(\mathrm{fr}=\mathrm{f} / \mathrm{F} \times 100)$ and $(\mathrm{b})$ the relative distance $\mathrm{d} r$ between two microirradiated nuclear sites $(\mathrm{dr}=\mathrm{d} / \mathrm{D})$. In the case when the chromosome arrangement remained fixed during interphase we expected constant values for fr and $\mathrm{dr}$ independent of changes of the nuclear size. Frequency distribution curves for fr-values pooled from all experiments are shown in Fig. 7. With increasing postincubation period these curves showed a shift to larger fr-values. The increase of $\mathrm{fr}$ with time was found to be significant by linear regression analysis $(P<0.001)$ with a regression coefficient of +0.20 . Table 2 shows the average fr-values obtained for
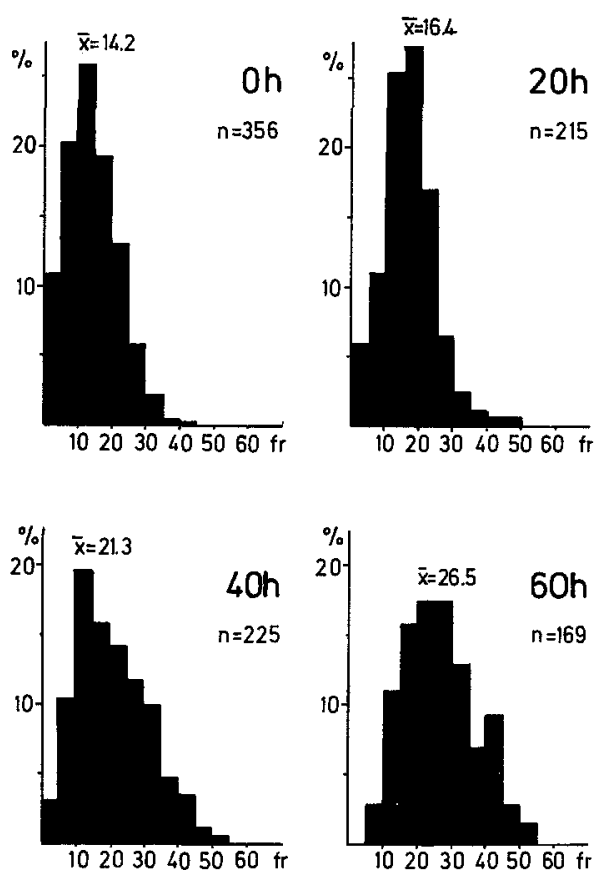

Fig. 7. Frequency distribution of UDS-labelled area fr (compare Fig. 6). Nuclei were microirradiated at one site in G1, pulse-labelled with ${ }^{3} \mathrm{H}-$ thymidine for $2 \mathrm{~h}$ and processed for autoradiography either immediately thereafter $(0 \mathrm{~h})$ or after 20,40 , and $60 \mathrm{~h}$ postincubation with fresh medium with $15 \%$ FCS. Nuclei were grouped into subclasses according to fr-values. In subclass $0-5$ the smallest fr-value was $3 \%$. Ordinate: Relative frequency for each subclass as percent of total number $n$ of evaluated nuclei. Abscissa: $\mathrm{fr}=\mathrm{f} / \mathrm{F} \times 100$, i.e. labelled area $\mathrm{f}$ as percent of total nuclear area $\mathrm{F}$. Linear regression analysis shows a significant increase $(P<0.001)$ of fr with increasing postincubation period. For further details of measurements compare Table 2

Table 2. UDS-labelled nuclear area fr ( $=$ percent of total nuclear area) resulting from microirradiation of a single nuclear site in G1: Effect of irradiation time and postincubation time after pulse-labelling with ${ }^{3} \mathrm{H}$-thymidine. For further explanation see Fig. 6

\begin{tabular}{|c|c|c|c|c|c|c|}
\hline Irradiation time & \multicolumn{6}{|c|}{ Postincubation time } \\
\hline $1 / 125 \mathrm{~s}$ & $\begin{array}{l}13.8 \pm 0.60 \\
(n=159)\end{array}$ & - & $\begin{array}{l}19.1 \pm 0.77 \\
(n=91)\end{array}$ & - & $\begin{array}{l}23.8 \pm 1.00 \\
(n=117)\end{array}$ & $\begin{array}{l}27.9 \pm 1.06 \\
(n=83)\end{array}$ \\
\hline $1 / 60 s$ & $\begin{array}{l}11.3 \pm 1.13 \\
(n=21)\end{array}$ & $\begin{array}{l}11.1 \pm 1.10 \\
(n=36)\end{array}$ & $\begin{array}{l}10.7 \pm 0.88 \\
(n=46)\end{array}$ & $\begin{array}{l}12.5 \pm 0.84 \\
(n=34)\end{array}$ & - & - \\
\hline Pooled data (compare Fig. 7) & $\begin{array}{l}14.2 \pm 0.40 \\
(n=356)\end{array}$ & - & $\begin{array}{l}16.4 \pm 0.53 \\
(n=215)\end{array}$ & - & $\begin{array}{l}21.3 \pm 0.71 \\
(n=225)\end{array}$ & $\begin{array}{l}26.5 \pm 0.81 \\
(n=169)\end{array}$ \\
\hline
\end{tabular}

For each experiment the average fr and the standard error of the mean (SEM) are indicated $n$ : Number of evaluated nuclei 
different UV-doses and postincubation periods. The increase of fr was most pronounced at the lowest UV-dose (1/125s). Here, the average fr-value had roughly doubled after $60 \mathrm{~h}$ and significant differences were obtained by $t$-test between 0 and $20 \mathrm{~h}$ $(P<0.001), 20$ and $40 \mathrm{~h}(P<0.001)$, and 40 and $60 \mathrm{~h}(P<0.01)$. A somewhat smaller increase of $f r$ at higher doses might indicate a dose dependent inhibition of microirradiation on chromatin movements. The results of measurements of $\mathrm{dr}$ between two microirradiated nuclear sites are shown in Table 3 and Fig. 8. Linear regression analysis of pooled data showed a slight but significant $(P<0.001)$ decrease of $\mathrm{dr}$ with increasing postincubation time (regression coefficient -0.0015 ), while the absolute distance $\mathrm{d}$ between the spots remained roughly constant.

Chromosome Arrangement at the Nuclear Edge. Since the nucleus of Chinese hamster fibroblasts resembles a flat ellipsoid the nuclear edge can be specified as the region where the curvature of the nuclear envelope has its maximum. The question arises whether particular chromosomal regions, e.g. telomers, are preferentially associated with this region. In the following experiments the periphery of G1-nuclei including the nuclear edge was microirradiated. Microirradiated cells were pulse-labelled with ${ }^{3} \mathrm{H}$-thymidine as described above. Thereafter cells were fixed and processed for autoradiography either immediately (Fig.9) or metaphase spreads were obtained from microirradiated cells after an additional period of growth for some 30 to $40 \mathrm{~h}$. Spreads were then stained with DAPI, photographed with a Zeiss fluorescence microscope III, and examined for the distribution of UDSlabel after autoradiography (Fig. 10). This approach was limited by the small number of UDS-labelled cells which entered the first postirradiation mitosis, but in a total of nine spreads labelled chromosomes could be identified by their morphology and banding patterns. The average number of labelled chromosomes per spread was 4.3 (range 1-7). Some of these chromosomes were damaged, but the majority appeared intact. UDS-label was clearly distinguishable from label patterns which originate from semiconservative DNA-synthesis by criteria previously described (Zorn et al. 1979). Notably, labelled chromosomes lay fairly adjacent to each other (Fig. 10). In 31 of a total of 39 labelled chromosomes the respective homologue was unlabelled. In four cases concerning chromosomes No.1, 2, 3, and 5, both homologues were jointly labelled. The question of random or non-random neighbourhood relationships between territories of

Table 3. Relative distance $d r$ between two UDS-labelled sites resulting from microirradiation of two nuclear sites in G1: Effect of irradiation time and postincubation time after pulse-labelling with ${ }^{3} \mathrm{H}$-thymidine. For further explanation see Fig. 6

\begin{tabular}{|c|c|c|c|c|}
\hline Irradiation time & \multicolumn{4}{|c|}{ Postincubation period } \\
\hline $1 / 125-1 / 30 \mathrm{~s}$ & $\begin{array}{l}0.64 \pm 0.011 \\
(n=50)\end{array}$ & $\begin{array}{l}0.64 \pm 0.012 \\
(n=46)\end{array}$ & $\begin{array}{l}0.63 \pm 0.015 \\
(n=23)\end{array}$ & $\begin{array}{l}0.59 \pm 0.020 \\
(n=38)\end{array}$ \\
\hline $1 / 15 \mathrm{~s}$ & $\begin{array}{l}0.66 \pm 0.015 \\
(n=30)\end{array}$ & $\begin{array}{l}0.60 \pm 0.019 \\
(n=34) \\
\end{array}$ & - & $\begin{array}{l}0.58 \pm 0.016 \\
(n=32)\end{array}$ \\
\hline
\end{tabular}

For each experiment the average $\mathrm{dr}$ and the standard error of the mean (SEM) are indicated

$n$ : Number of evaluated nuclei

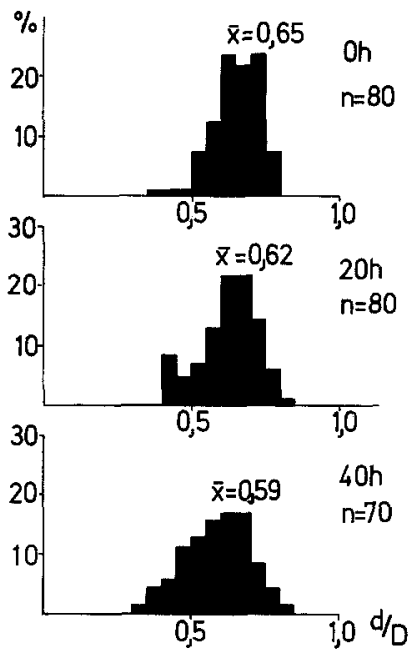

Fig. 8. Relative distance dr (compare Fig. 6) between two microirradiated nuclear sites. After microirradiation in G1 the cells were labelled with ${ }^{3} \mathrm{H}$-thymidine for $2 \mathrm{~h}$ and processed for autoradiography either immediately thereafter or after 20 and $40 \mathrm{~h}$ of additional growth. Nuclei were arbitrarily grouped into subclasses according to dr-values. The ordinate shows the relative frequency (\% of total number $n$ of evaluated nuclei) for each subclass. Linear regression analysis shows a significant $(P<0.001)$ albeit small decrease of $\mathrm{dr}$ with increasing postincubation time. For further details of measurements compare Table 3 homologous chromosomes will be discussed in a subsequent paper (Cremer et al. in preparation).

With regard to UDS-label patterns on individual chromosomes several features are of interest. Three small chromosomes appeared labelled over their whole length, while 36 chromosomes showed label only over a part of varying length. This result can easily be explained by the chance localization of the microbeam between the borders of several territories. Six chromosomes (No.1, three cases, No.2, 3, 5, one case each) showed joint labelling of both their distal chromosome arms, while their proximal arm and the centromeric region was not labelled (see Fig. 10 for examples). This result fits with a Vshaped orientation of these chromosomes in the interphase nucleus with their telomeres situated adjacent to each other at or close to the nuclear edge. The average frequency with which such a label pattern can be expected would depend on the angle between the two chromosome arms of V-shaped chromosomes and on the diameter of the microbeam. Present microbeam data are not sufficient to decide whether the V-shaped orientation of metacentric and submetacentric chromosomes is the rule or rather an exception in the Chinese hamster cell nucleus. Labelled chromosome parts included a total of 15 centromeric and 39 telomeric regions, one labelled telomeric region in 21 chromosomes and two in 9 chromosomes. In 7 chromosomes the centromere and one telomere were jointly included in the labelled part. Only 


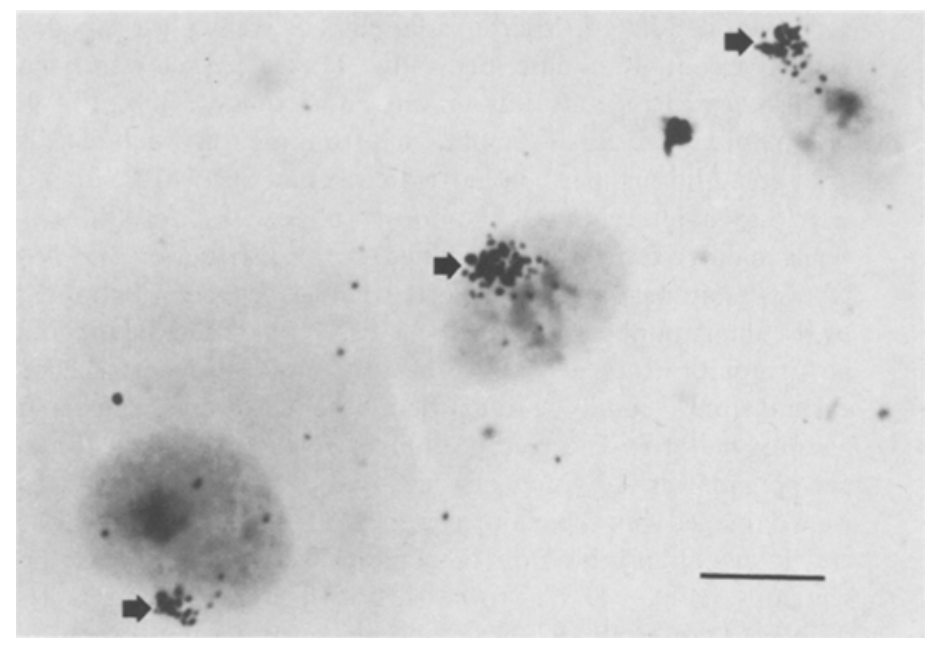

Fig. 9. Autoradiographs of three cells after UV-microirradiation of the nuclear edge, labelling with ${ }^{3} \mathrm{H}$-thymidine for $2 \mathrm{~h}$ and fixation immediately after the label period. Bar denotes $10 \mu \mathrm{m}$
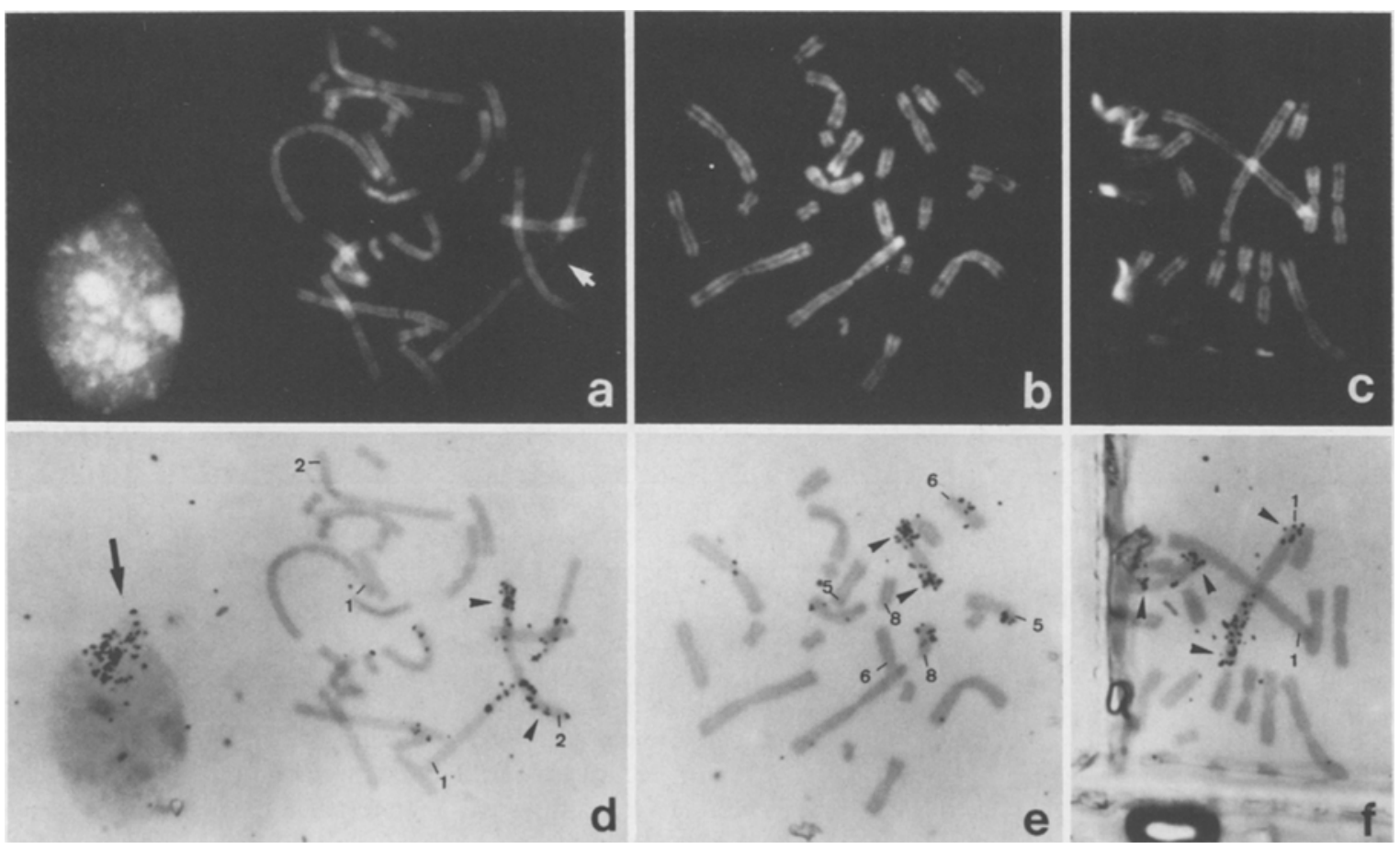

Fig. 10a-f. Cells were microirradiated at one site of the nuclear edge in G1 (compare Fig. 9) and pulse-labelled with ${ }^{3} \mathrm{H}$-thymidine for two hours. Chromosome preparations were performed in situ after a postincubation period of $30-40 \mathrm{~h}$ and stained with DAPI. a-c Fluorescence microscopy of three metaphase spreads; $\mathbf{d}-\mathbf{f}$ autoradiographs of the same metaphase spreads. UDS-labelled chromosome segments were associated with each other in G1 at the microirradiated part of the nuclear edge. Small arrows point to chromosomes in which distal regions of both arms are jointly labelled. Labelled chromosomes and their respective homologues are designated by their number in cases where identification was possible. Miscondensation within the labelled region of a chromosome no. I may be noted (Fig. 10a, white arrow). The large arrow in Fig. $10 \mathrm{~d}$ indicates UDS-label still localized at the edge of an interphase nucleus some $40 \mathrm{~h}$ after microirradiation

in 4 cases label appeared clearly restricted to a part of chromosome arms, while the centromeric and telomeric regions were not involved.

\section{Discussion}

In the present investigation we have applied two different approaches to investigate the internal arrangement of chromosomes in the interphase nucleus of Chinese hamster fibroblasts, namely premature chromosome condensation and UV-microirradiation. In spite of the inherent limitations of both these approaches, which will be discussed below, the results support several of Rabl's $(1885)$ and Boveri's $(1888,1909)$ predictions namely: (1) that the relative positions of chromosome territories in the interphase nucleus which are formed in telophase are preserved during the subsequent interphase and (2) that chromosomes retain their anaphase-telophase orientation during interphase.

The small number of UDS-labelled chromosomes per metaphase which has been obtained after microirradiation and pulse-labelling of the nucleus with ${ }^{3} \mathrm{H}$-thymidine in $\mathrm{Gl}$ strongly supports the concept of a territorial organization of chromosomes in the interphase nucleus at this stage of the cell cycle 
(Cremer et al. 1979a; Zorn et al. 1979; this paper). Furthermore, use of antibodies specific for the detection of UVirradiated DNA has made it possible to detect microirradiated chromatin by indirect immunofluorescence microscopy both in microirradiated interphase nuclei and in metaphases obtained from cells after microirradiation of the nucleus at any stage of the preceding interphase (Cremer et al. 1980; Hens et al. in preparation). In particular, we have observed by indirect immunofluorescence microscopy specific staining of only a very few chromosomal segments in metaphase after microirradiation of the nucleus in S-phase. These findings show that the territorial arrangement of chromosomes as previously demonstrated for the G1-nucleus, is also maintained in the subsequent S-phase (Hens et al. in preparation).

Interpretation of fr- and dr-values as parameters of possible changes in the relative positions of chromosome territories during the cell cycle (Figs. 6-8; Tables 2 and 3) should be performed with the reservation in mind that microirradiation might induce changes in the condensation of damaged chromatin and affect the mobility of the microirradiated chromosomes in the interphase nucleus to a certain extent. Obstacles against movements of microirradiated chromatin might result from microirradiation induced "stickiness" and/or interchanges between microirradiated chromosomes. On the other hand, UDS-label patterns on individual chromosomes indicate that the microbeam had affected only a part of most irradiated chromosomes. Accordingly, major movements of the nonirradiated part of these chromosomes and of non-irradiated neighbouring chromosomes should have grossly influenced the interphase topography of the microirradiated chromatin as well. Time dependent alterations of fr- and dr-values, however, while statistically significant were relatively small, although progress in the cell cycle from G1 into S-phase was demonstrated for the majority of microirradiated cells (Fig. 5). Even after $60 \mathrm{~h}$ postincubation the labelled nuclear region appeared to be still coherent. In the case of microirradiation of the nuclear edge or nuclear poles the localization of the labelled areas remained at these sites even after $40 \mathrm{~h}$. In a preliminary series of experiments (Cremer et al. 1979 b) microirradiation of the G1-nucleus was restricted to the smallest nuclear area possible. Pulse-labelling with ${ }^{3} \mathrm{H}$-thymidine was performed under slightly modified conditions and resulted in fr-values of $3-4 \%$ in cells fixed immediately after the labelling period and of $4-5 \%$ in cells fixed after additional growth periods up to $80 \mathrm{~h}$. In conclusion, our data do not indicate major changes of the arrangement of interphase chromosomes when the cells proceed from G1 into S-phase, while small time dependent changes of the nuclear space and the exact nuclear position occupied by single interphase chromosomes or chromosome segments would be compatible with these data. A rather static arrangement of chromosome territories during interphase would be the logical consequence of many fixing points of chromatin to the nuclear matrix and nuclear envelope. Slight changes of chromatin distribution in microirradiated nuclei support the idea that this fixation is not completely rigid. Either fixing points might be resolved and newly formed at a somewhat distant site or chromatin fibres might slide along certain fixing points (Pardoll et al. 1980). Such a dynamic association of chromatin fibres with the nuclear matrix might be of great functional importance but does not seem to influence the territorial organization of interphase chromosomes and their gross arrangement in general.
In the light of the above conclusions the Rabl-type polarization of G1 and G2 PCCs appears as a passive relic of their anaphase-telophase orientation, which is maintained throughout the cell cycle. Loss of polarization, which was observed in many Chinese hamster PCCs ranging from $28 \%$ to $72 \%$ in different experiments, is most likely due to artefacts of the PCC preparation procedure, including hypotonic treatment and spreading of PCC chromosomes. It has previously been shown that in Muntjac cells $(2 n=7)$ the frequency of completely polarized PCCs can be as high as $84 \%$, the remainder showing partial polarization (Sperling and Luedtke 1981). Moreover, even Muntjac $\mathrm{G}_{0}$ lymphocytes which may be arrested at this stage for up to several months or years exhibit this polarized orientation in most of the cells analyzed (Sperling and Luedtke 1981). In Chinese hamster cells $(2 n=22)$ the maximum frequency of completely polarized PCCs was only $36 \%$. Probably a higher number of chromosomes decreases the chance to preserve the Rabl-type orientation during the PCC-procedure (Sperling 1982).

On the other hand the possibility should be taken into consideration that PCC might initiate movements of chromosomes similar to those which start in prophase. Coorientation of centromeres in PCCs might then in part reflect some progression of the centromeric regions of chromosomes towards each other after the induction of PCC. Microirradiation of the periphery of the nucleus including the nuclear edge more often resulted in labelling of the distal arms of chromosomes, including the telomeres, than in labelling of centromeric regions. However, when we take into account that the number of telomeres exceeds the number of centromeres our present data do not show a remarkable excess of labelled telomeres in the microirradiated nuclear part. Visualization of centromeres in the intact nucleus has become possible by indirect immunofluorescence microscopy with antibodies which bind specifically to centromeres (Moroi et al. 1980). Preliminary data seem to indicate that the centromeres of Chinese hamster cells are clustered in some nuclei but distributed more or less over the whole nuclear area in others (Moroi et al. 1980). Further discussion of the distribution of centromeres and telomeres in the Chinese hamster cell nucleus is postponed until more data become available.

Acknowledgements. This work has been supported by the Deutsche Forschungsgemeinschaft. We thank Drs. T. M. Schroeder-Kurth and F. Vogel (Heidelberg) for stimulating discussions, Dr. U. Wolf (Freiburg i. Br.) for his continuous interest and support, and Dipl. Math. J. Krüger (Heidelberg) for advice in statistical analysis of the data.

\section{References}

Avivi L, Feldman M (1980) Arrangement of chromosomes in the interphase nucleus of plants. Hum Genet 55:281-295

Berezney R, Buchholtz LA (1981) Dynamic association of replicating DNA fragments with the nuclear matrix of regenerating liver. Exp Cell Res 132:1-13

Boveri Th (1888) Zellen Studien. Jena, Z Naturw 22:687-882

Boveri Th (1909) Die Blastomerenkerne von Ascaris megalocephala und die Theorie der Chromosomenindividualität. Arch Zellforsch 3: $181-268$

Cremer C, Zorn C, Cremer T (1974) An ultraviolet microbeam for $257 \mathrm{~nm}$. Microsc Acta 75:331-337

Cremer C, Cremer T, Zorn C, Schoeller L (1976) Effects of laser-UVmicroirradiation $(\lambda=2573 \AA$ ) on proliferation of Chinese hamster cells. Radiat Res 66:106-121

Cremer C, Cremer T, Zorn C, Cioreanu V (1979a) Partial irradiation of Chinese hamster cell nuclei and detection of unscheduled DNA 
synthesis in interphase and metaphase. A tool to investigate the arrangement of interphase chromosomes in mammalian cells. Hoppe Seyler's Z Physiol Chem 360:244-245

Cremer T, Cremer C, Cioreanu V (1979b) Unscheduled DNA synthesis induced by laser-UV-microirradiation of the cell nucleus in G1 and autoradiographic examination of the distribution of microirradiated chromatin in Gl and S-phase. Eur J Cell Biol 20:127-128

Cremer C, Cremer T, Fukuda M, Nakanishi K (1980) Detection of laserUV-microirradiation induced DNA photolesions by immunofluorescent staining. Hum Genet 54:107-110

Cremer C, Cremer T, Jabbur G (1981) Laser-UV-microirradiation of Chinese hamster cells: The influence of the distribution of photolesions on unscheduled DNA synthesis. Photochem Photobiol 33: 925-928

Comings DE (1968) The rationale for an ordered arrangement of chromatin in the interphase nucleus. Am J Hum Genet 20:440-460

Comings DE (1980) Arrangement of chromatin in the nucleus. Hum Genet 53:131-143

Hager D, Schroeder TM, Vogel F (to be published) Position of chromosomes in the interphase nucleus. An analysis of nonhomologous interchanges in lymphocyte cultures after Trenimon treatment and from two patients with Fanconi's anemia and Bloom's syndrome. Hum Genet

Hens L, Kirsch-Volders M, Susanne C, Galperin-Lemaitre H (1975) Relative position of trypsin banded homologous chromosomes in human metaphase figures. Hum Genet $28: 303-311$

Hoehn H, Martin GM (1973) Nonrandom arrangement of human chromatin: topography of disomic markers $X, Y$, and $1 \mathrm{~h}+$. Cytogenet Cell Genet 12:443-452

Kirsch-Volders M, Hens L, Susanne C, Galperin-Lemaitre H (1977) Stability of centromere-center distances in normal human metaphases. Cytogenet Cell Genet 18:61-74

Kirsch-Volders M, Hens L, Susanne C (1980) Telomere and centromere association tendencies in the human male metaphase complement. Hum Genet 54:69-77

Moroi Y, Peebles C, Fritzler MJ, Steigerwald J, Tan EM (1980) Autoantibody to centromere (kinetochore) in scleroderma sera. Proc Natl Acad Sci USA 77:1627-1631
Pardoll DM, Vogelstein B, Coffey DS (1980) A fixed site of DNA replication in eucaryotic cells. Cell 19:527-536

Rabl C (1885) Über Zelltheilung. Morphologisches Jahrbuch. Gegenbaur C (ed) 10:214-330

Schnedl W, Mikelsaar A-V, Breitenbach M, Dann O (1977) DIPI and DAPI: fluorescence banding with only negligible fading. Hum Genet $36: 167-172$

Sperling K, Rao PN (1974) The phenomenon of premature chromosome condensation: its relevance to basic and applied research. Hum Genet 23:235-258

Sperling K, Luedtke E-K (1981) Arrangement of prematurely condensed chromosomes in cultured cells and lymphocytes of the Indian Muntjac. Chromosoma 83:541-553

Sperling K (1982) Cell cycle and chromosome cycle: morphological and functional aspects. In: Rao PN, Johnson RT, Sperling K (eds) Premature chromosome condensation: application in basic, mutation and clinical research. Academic Press, New York

Vogel F, Schroeder TM (1974) The internal order of the interphase nucleus. Hum Genet 25:265-297

Zang KD, Back E (1968) Quantitative studies of the arrangement of human chromosomes. I. Individual features in the association pattern of acrocentric chromosomes of normal males and females. Cytogenetic 7:455-470

Zankl H, Zang KD (1974) Quantitative studies on the arrangement of human metaphase chromosomes. IV. The association frequerncy of human acrocentric marker chromosomes. Hum Genet 23:259-265

Zorn C, Cremer T, Cremer C, Zimmer J (1976) Laser-UV-microirradiation of interphase nuclei and posttreatment with caffeine: a new approach to establish the arrangement of interphase chromosomes. Hum Genet 35:83-89

Zorn C, Cremer C, Cremer T, Zimmer J (1979) Unscheduled DNA synthesis after partial UV-irradiation of the cell nucleus. Distribution in interphase and metaphase. Exp Cell Res 124:111-119

Received September 22, 1981 